\title{
Validation of the Octavius 4D Measuring System In Verifying Advanced External Beams Radiotherapy Techniques
}

\author{
A.E. Amin, A. M. Sallam*, E. M. El-Sayed", S.M. El-Sayed and N.R. Salem* \\ Radiation Oncology and Nuclear Medicine Department, Faculty of Medicine, Ain \\ Shams University, and *Biophysics branch, Department of Physics, Faculty of Science, \\ Ain Shams University, Cairo, Egypt.
}

\begin{abstract}
$\mathbf{T}$ HE DEVELOPMENT of advanced techniques as Intensity modulated radiotherapy (IMRT) and volumetric modulated arc therapy (VMAT) involves computer controlled dynamic MLC motion and results in numerous steep dose gradients. Thus specific quality assurance (QA) became essential requirement to meet this complexity. The conventional dosimetery techniques, such as ionization chamber, point dose measurements and film dosimetry, are gradually being replaced by detector arrays which can produce accurate results immediately. There are many detector arrays available in the Market. We have used one of these commercial systems named Octavius $4 \mathrm{D}$. We analyzed the warming behavior of our detector array. We studied the dose and dose rate linearity. We assessed the response of the detectors to the change in field size. The OCTAVIUS phantom rotates synchronously with the gantry, taking time- and gantry angle-resolved dose measurements. The accuracy of the synchronizing motion of the phantom was validated. We performed the verification of 26 IMRT \& VMAT plans of different cases (head and neck, brain, breast and prostate) using the common criteria $3 \% / 3 \mathrm{~mm}$ and passing threshold rate $>95 \%$. We tested the sensitivity of our QA system and investigated the factors that might affect its performance. It was found that the detector array response is stable after warming up with $800 \mathrm{MU}$, the dose rate measurements and the dose response is linear with $\mathrm{R} 2=0.929$ and the dose response of the output factors shows a similar trend. The Octavius phantom was found to be moving in a synchronizing motion. The gamma index for the clinical IMRT plans were found to be $93.3 \%, 98.0 \%, 95.5 \%$ and $96.2 \%$ for head and neck, prostate, breast and brain cases, and for VMAT plans are 96.3\%, 99.4 \%, 98.1\% and $98.6 \%$ for head and neck, prostate, breast and brain, respectively, using gamma criteria $3 \% / 3 \mathrm{~mm}$. The aim of this work is to characterize and validate the Octavius $4 \mathrm{D}$ detector array. We also aim at evaluating this system for dose measurement of our VMAT and IMRT treatment plans.
\end{abstract}

Keywords: Octavius 4D, VMAT, IMRT, Arrays, Verification, Synchronizing, Gamma and Passing rate.

The advanced external beams radiotherapy techniques as intensity modulated radiotherapy (IMRT) and Volumetric-modulated arc therapy (VMAT) have facilitated complex treatments that achieves optimum conformal dose to the target volume whilst minimizing the dose to surrounding normal tissue. IMRT utilizes step and shoot or dynamic sliding window multileaf collimators (MLC) to deliver non uniform fluence to the patient from any given position of the treatment beam to optimize the composite dose distribution.
VMAT combines the dynamic MLC delivery with the modulation of gantry speed and dose rate. Thus IMRT and VMAT give superior treatment plans if compared to the conventional techniques. The 3D dose distribution in IMRT and VMAT plans is characterized by steep and numerous dose gradients which is related to the complex MLC pattern. As the treatment complexity increases, the inaccuracy, uncertainty and the error in dose delivery increases which lead to potential clinical implications, $(2,3)$ so, these external advanced

\#Correspondsing author: rnoha2020@yahoo.com

DOI :10.21608/ejbbe.2018.2295.1010

(C)The Nathional Information and Documentation Center (NIDOC) 
techniques require a specific quality assurance. Three-Dimensional conformal radiotherapy (3DCRT) treatment is delivered via static fields that conform as closely as possible to the target volume as seen from the beam eye view. The MLC will be shaped in accordance with the target at the different gantry angles. There is no modulation of the dose rate and there is no optimized intensity modulation of the beam with any gantry rotation. Thus treatment plans for 3DCRT will show a uniform dose distribution inside the target volume. This dose uniformity inside the target is dependent on the uniformity of our treatment beams which can be easily verified within the weekly routine check, measuring the flatness and symmetry using our conventional measuring techniques ${ }^{(4)}$. Traditionally the verification of IMRT \& VMAT has been done using ionization chambers and film within cubic or semi-anthropomorphic phantoms. Once an IMRT and VMAT treatment plan for a patient is complete, a verification plan is created using the treatment planning systems. Essentially, a verification plan is a copy of the same gantry angles, dynamic MLC pattern, and monitor units of the clinical plan and it is calculated on a CT scan of the physical phantom which will be used in performing the verification measurement $\mathrm{A}$ dose image is generated from this verification plan at the level of the detector array and is then compared with measurement ${ }^{(5)}$. As with the more use of IMRT and with the more complex deliveries like VMAT, quality assurance (QA) has developed quickly to meet the demand for more efficient techniques for treatment plans verification. ${ }^{(6,}$ ${ }^{7}$ In recent years, various commercial $2 \mathrm{D}$ and 3D ionization chamber or diode detector arrays have become available, allowing for verification of absolute dose with immediate results. Conventional methods, such as ionization chamber point dose measurements and film dosimetry, are gradually being replaced by detector arrays. These devices have allowed centers to simplify and organize their QA and increase the number of patients treated with IMRT and VMAT. However, detector arrays are limited by their resolution, giving rise to concerns about their sensitivity to errors $^{(4)}$. There are many commercial detector arrays like the Delta4 (ScandiDos AB, Uppsala, Sweden), ArcCHECK/MapCHECK (Sun Nuclear Corp., Melbourne, FL), MatriXX (IBA Dosimetry GmbH, Schwarzenbruck, Germany) and 2D-ARRAY seven29 (PTW, Freiburg, Germany). many papers have been previously published to study the sensitivity to IMRT and VMAT for the
$\operatorname{Delta}^{(8,9,10)}, \operatorname{ArcCHECK}^{(9,11)}$ and MapCHECK $^{(12,13)}$ .The PTW Array 729 (PTW,Freiburg, Germany) with the stationary hexagonal homogenous Octavius phantom have been employed clinically to verify the IMRT and the rotational delivery ${ }^{(14,15)}$. The main limitations of the 2D detectors array are the resolution of the detector size, spacing, ${ }^{(16)}$ and the directional dependence of the detectors ${ }^{(17)}$. Directional dependence can result in differences in detector response of up to $15 \%$ between different gantry angles, ${ }^{(17)}$ until the development of the cylindrical Octavius phantom with its detector array. The latest version of the 2D detector array composed of a matrix of 729 ionization chambers can be placed inside the cylindrical Octavius 4D phantom. The Octavius 4D phantom is connected to an inclinometer placed on the gantry of the linear accelerator allowing the phantom to detect the different gantry angles of different fields for the IMRT or VMAT plans. The Octavius 4D measuring system rotates synchronously with the gantry to insure that the detector array is always perpendicular to the incident beam measuring a dose plane for each gantry angle. And hence avoids any directional dependence. The Octavius system then utilizes a reconstruction algorithm to construct the $3 \mathrm{D}$ dose. This dose is then compared to that calculated using the treatment planning system (TPS). Conor et al ${ }^{(18)}$ have studied the Octavius $4 \mathrm{D}$ measuring system together with the 729 detector array. They have characterized their system for flattening filter (FF) and flattening filter free (FFF) static and rotational beams. The device was assessed for verification with FF and FFF RapidArc treatment plans. They concluded that the Octavius 4D phantom with associated Octavius detector 729 ionization chamber array is a dosimetrically and mechanically stable device for pretreatment verification of $\mathrm{FF}$ and FFF RapidArc treatments. Further improvements may be possible through use of a detector array with higher spatial resolution. Calvo et al ${ }^{(19)}$ evaluated the 3D dose reconstruction of pretreatment verification plans using multiple $2 \mathrm{D}$ planes acquired from the OCTAVIUS phantom and the Seven29 detector array. They concluded that a simple cylindrical geometry-based, linear interpolation method is able to predict good agreement in the high dose region between the reconstructed volumetric dose and the planned volumetric dose. It is important to mention that the interpolation algorithm introduces dose discrepancies in small regions within the high dose gradients due to the interpolation itself. They 
also stated that the work they presented serves as a good starting point to establish a benchmark for the level of manipulation necessary to obtain 3D dose delivery quality assurance using current technology. From the above introduction, it is clear that investigating the performance of the QA system is necessary to insure accurate delivery of the radiotherapy treatment of cancer patients. In this study we aim to characterize our Octavius 4D detector array QA system. We will also evaluate this system for dose measurement of our VMAT and IMRT treatment planes.

\section{Materials and Methods}

The Octavius 4D measuring system is a combination of a phantom and detector array. The OCTAVIUS Detector 729 T10040 and the Detector Interface $4000 \mathrm{~T} 16039$ form a multi-channel dosimeter for dose and dose rate measurement in radiotherapy. The OCTAVIUS Detector 729 (PTW, Freiburg, Germany) is a 2-dimensional detector array with 729 (27 $\mathrm{x} 27 \mathrm{~cm}^{2}$ ) equally spaced vented ionization chambers with a space between one and another is $1 \mathrm{~cm}^{2}$ and each chamber has a size of $0.5 \times 0.5 \times$ $0.5 \mathrm{~cm}^{3}$. The Detector Interface $4000 \mathrm{~T} 16039$ is the interface between the OCTAVIUS Detector 729 and the controlling measuring software. The physical dimensions of the Octavius detector 729 are $2.2 \mathrm{~cm}$ (thickness) $\times 30.0 \mathrm{~cm}$ (width) $\times 42.0$ $\mathrm{cm}$ (length) with the effective reference point located $0.75 \mathrm{~cm}$ below the surface of the array. The previous versions of this detector had a doserate measurement range of $0.5-10 \mathrm{~Gy} / \mathrm{min},{ }^{(13)}$ but the new array provides a higher dose rate range of $0.5-48 \mathrm{~Gy} / \mathrm{min}$. The detector dose resolution is 1 $\mathrm{mGy}$ and there is no upper limit specified for dose delivery. The OCTAVIUS Rotation Unit T40056 is a cylindrical phantom with a diameter $32.0 \mathrm{~cm}$, length $34.3 \mathrm{~cm}$ and the angular range is $\pm 360^{\circ}$ this phantom has a slot for insertion of the detector array. The built-in mechanics and the motor allow the cylinder to rotate, ensuring perpendicular incidence of the radiation beam on the detector array at all times. Rotation direction and speed are controlled by means of the Inclinometer T43035 and the OCTAVIUS control Unit T40058.All the measurements are applied on a Unique linear accelerator with a various dose rate from $100 \mathrm{MU} /$ $\min$ to $600 \mathrm{Mu} / \mathrm{min}$ according to the experiments and the clinical IMRT and VMAT plans. Figure 1 show the setup of the Octavius system and the detector array is inserted and its isocenter is defined by the room laser. Eclipse planning system was used to calculate 26 treatment plans for different body sites including prostate, breast, brain and head and neck cases. Eclipse utilizes the PO optimization algorithm for IMRT optimization and the analytical anisotropic algorithm (AAA) for the final dose calculation. IMRT and VMAT plans were optimized using photon optimization algorithm PO and the final dose calculation used AAA. The percentage depth dose (PDD) curves for $6 \mathrm{MV}$ photons were used within the VeriSoft 5.1 analysis software (PTW, Freiburg, Germany) for the reconstruction of each plane dose for every gantry angle and was matched with the calculated matrix dose .Verisoft software allows a 2D gamma analysis for comparing planes or 3D gamma analysis ${ }^{(20)}$ for comparing measured and calculated volumes. Throughout this work the global 3D gamma analysis was employed with criterion $3 \% / 3 \mathrm{~mm}, 10 \%$ minimum threshold and the threshold passing value is $>95 \%$.

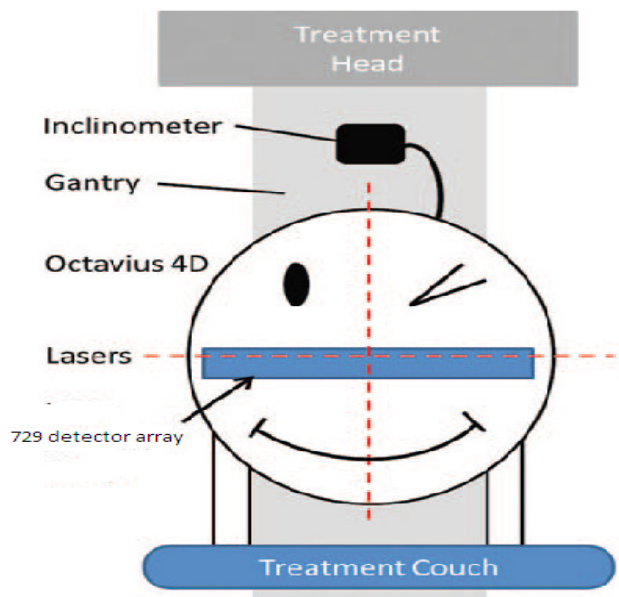

Fig. 1: Set up of the Octavius 4 D measuring system on the linac. The inclinometer attached to the head of gantry allowing the phantom to rotate in a synchronizing motion with gantry angle making the detector array which inserted in the phantom slot to be perpendicular on the incident beam. The isocenter of the phantom is defined by the room laser.

Characterization of the 729-detector array for 6MV.

Warm up

The warm up of the ionization chambers is necessary ${ }^{(21,22)}$ to ensure the stability of the detector array before starting the pretreatment dosimetric verification. To determine the number of monitor units required for warm up of the 
detector array. The ionization chambers within the detector array inserted in the Octavius phantom were irradiated with a static $27 \times 27 \mathrm{~cm}^{2}$ beam using $100 \mathrm{MUs} 15$ times. The dose response of the central ionization chamber on each occasion was analyzed .Each measurement was normalized to the 15 th measurement.

\section{Dose rate linearity}

To study the dose rate linearity of the detector array, it was irradiated with $100 \mathrm{MU}$ using a $6 \mathrm{MV}$ $10 \times 10 \mathrm{~cm}^{2}$ static field using different dose rates between 100 to $600 \mathrm{MU} / \mathrm{min}$. The dose response of the central ionization chamber of the array was analyzed for the different dose rates and then measured dose was normalized to the nominal standard dose rate (400 MU/min).

\section{Dose linearity}

We started this test with 1 MU which delivers 0.007 Gy to our detector. We selected this value as it is higher than the detector sensitivity which is $0.001 \mathrm{~Gy}$.The dose linearity of the detector array was tested by irradiating the detector with static field $10 \times 10 \mathrm{~cm}^{2}$ with doses between $1 \mathrm{MU}$ (0.07Gy) - 500MU (3.151Gy) .The dose was analyzed at the central ionization chamber of the detector array for each delivered monitor unit and then normalized to the output $100 \mathrm{MU}(0.628 \mathrm{~Gy})$.

\section{Output factors (field size)}

The response of the detector array as a function of field sizes(output factors) was investigated by delivering $6 \mathrm{MV}$ static square fields $1 \times 1 \mathrm{~cm}^{2}$ to $27 \times 27 \mathrm{~cm}^{2}$ with $100 \mathrm{MU}$ to the detector array. The dose outputs for each field size was analyzed at the central ionization chamber of the detector and normalized to the $10 \times 10 \mathrm{~cm}^{2}$ static field.

\section{The accuracy of the synchronizing motion}

The OCTAVIUS phantom T40056 which we used is cylindrical phantom, rotating in a synchronizing motion with the gantry angle, allowing each incident beam with specific angle to be perpendicular to the detector. This omit any directional dependence for the detectors array as it can result in differences in detector response of up to $15 \%$ between different gantry angles ${ }^{(17)}$. To test the accuracy of this synchronizing motion, we used $6 \mathrm{MV}$ with $10 \times 10 \mathrm{~cm}^{2}$ field size that was delivered in half arc starting from $90^{\circ}$ and rotating clockwise till ending at $270^{\circ}$. This half arc was used to avoid any beams passing through the couch. Then this $10 \times 10 \mathrm{~cm}^{2}$ half arc beam was reconstructed to an anterior static field with the same parameters of the arc (MU, MLC shape and length)but with gantry angle zero, removing the inclinometer data and verified using the Octavius phantom to calculate the gamma index. The gamma criteria used to calculate the gamma index is $3 \% / 3 \mathrm{~mm}$. We compared gamma index values of both the arc field and the static field.

\section{The reconstruction algorithm accuracy}

The Octavius 4D algorithm is based on the phantom density value on TPS and the percentage depth dose curves (PDD) that are used to reconstruct the dose on the line that connect detector and focused beam. To estimate the accuracy of the defined algorithm, we used a $10 \times 10 \mathrm{~cm}^{2}$ anterior static field with $100 \mathrm{MU}$ to be delivered to Linac and dosimetrically verified using the Octavius 4D measuring system to calculate the gamma index. The common criteria used is $3 \% / 3$ mmand the passing rate threshold is $95 \%$.

\section{Clinical IMRT and VMAT plans delivery}

A series of 26 IMRT and VMAT plans of different cases with different sites (prostate, breast, Head and Neck and brain) were calculated using the eclipse treatment planning system (TPS) and verified on the Octavius phantom to produce the measured image which will be compared against the calculated one on the TPS. This comparison and analysis was performed using the software Verisoft v5.1.The common criteria used is a $3 \% / 3$ $\mathrm{mm}$ and the passing rate threshold was $>95 \%$ to assess the treatment delivery. The gamma index was evaluated taking into account the $3 \mathrm{D}$ dose distribution, i.e. all the planes (coronal, sagittal and transverse), also the dose line profiles were analyzed using the same software.

\section{Test the sensitivity of the gamma criteria}

Since the IMRT plans of H\&N cases result in the least gamma passing rate using the common criteria $3 \% / 3 \mathrm{~mm}$ where the average of this gamma passing rate of IMRT plans for $\mathrm{H} \& \mathrm{~N}$ cases was $93.3 \%$ and this value couldn't achieve the threshold passing value $(>95 \%)$ ). so further analysis was done to IMRT plans of H\&N cases. We compared dose distribution calculated by eclipse treatment planning system to that measured by Octavius system using different criteria $(2 \% / 2 \mathrm{~mm}, 3 \% / 3 \mathrm{~mm}, 3 \% / 4 \mathrm{~mm}, 4 \% / 3 \mathrm{~mm}$ and $4 \% / 4 \mathrm{~mm}$ ), minimum threshold value is $10 \%$ and passing rate threshold value is $>95 \%$. 
Comparison between the stationary and rotational Octavius phantom measurements.

The IMRT plans of different cases were verified two times using the Octavius system. Firstly, allowing the Octavius phantom to be rotating and each delivered beam with different gantry angle is perpendicular to the detector array where the Octavius phantom define the degree of the angle to rotate through the inclinometer. Secondly, we removed the inclinometer data, allowed the Octavius phantom to be stationary and reconstructed all the plans to fixed angle zero. The gamma index was calculated for both states, using the common criteria $3 \% / 3 \mathrm{~mm}$. The calculated gamma index of the both cases were compared against each other.

\section{Factors affect the gamma index}

\section{The complexity}

The complexity in this study means, a plan with more than one target with different prescribed dose, showing a degree of dose gradient. To study how this complexity factor affect the gamma index, IMRT plan is calculated for this case three times using different prescribed dose for each plan, delivered to linac, verified by the Octavius verification system and the gamma index for each plan was calculated with Verisoft using the common criteria $3 \% / 3 \mathrm{~mm}$. The IMRT plans were calculated with prescription dose equal to 50Gy for the first plan, 50and 70 Gy for the second plan, and finally 50, 60 and 70 Gy for the third IMRT plan.

\section{Field sizes}

To study the effect of changing the field size on gamma index, anterior static fields of different field sizes ranging from $3 \times 3 \mathrm{~cm}^{2}$ to $30 \times 30 \mathrm{~cm}^{2}$ was used to deliver $100 \mathrm{MU}$ and then was verified by the Octavius 4D system. The gamma index was calculated for each different field size using the common criteria $3 \% / 3 \mathrm{~mm}$.

\section{Prescribed dose}

To study the effect of increasing the prescribed dose on gamma index, Anterior $10 \times 10 \mathrm{~cm}^{2}$ static field plans were calculated with different prescribed doses ranging from 50 to $600 \mathrm{cGy}$. The calculated dose distribution image of the TPS is compared against the measured dose distribution image by the Octavius system and the gamma index is calculated for each plan with its specific prescribed dose using the common criteria $3 \% / 3 \mathrm{~mm}$.

\section{The effect of collimation rotation}

To test the effect of collimation rotation, Plans were generated for an anterior $10 \times 10 \mathrm{~cm}^{2}$ static field, $10 \times 10 \mathrm{~cm}^{2}$ half arc $\left(90^{\circ}\right.$ to $\left.270^{\circ}\right)$ and $10 \times 10$ $\mathrm{cm}^{2}$ full arc $\left(181^{\circ}\right.$ to $\left.179^{\circ}\right)$ utilizing zero collimator angle. Then plans were regenerated utilizing $45^{\circ}$ collimator angle. All plans were verified by the Octavius system. Gamma index was calculated using the common criteria $3 \% / 3 \mathrm{~mm}$.

\section{Increasing number of fields}

The gamma index is calculated for a group of conformal plans with different number of fields of 3,5,7,9 and 11 fields using the common criteria $3 \% / 3 \mathrm{~mm}$.

\section{Results}

The $2 D$ array validation Warm up

The measured normalized doses by the detector array were plotted against the cumulative MUs figure 4.2 which shows that the normalized doses increase with the MUs up to $800 \mathrm{MU}$ and above $800 \mathrm{MU}$ the dose response is stable. This result indicates that a warm up of at least $800 \mathrm{MU}$ is required to ensure a stable detector response.

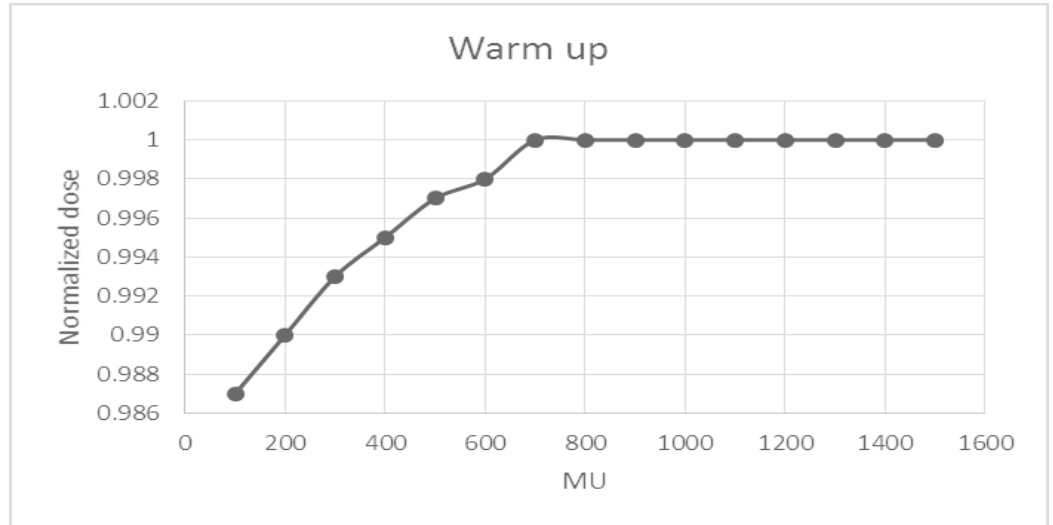

Fig.4.2 the normalized dose of the detector array during repeated irradiation of $100 \mathrm{MU}$. 
Dose rate linearity

In Figure 4.3 the normalized measured dose for the central ionization chamber of the detector array is plotted against the dose rate in the range
$100 \mathrm{MU} / \mathrm{min}(0.626 \mathrm{~Gy} / \mathrm{min})$ - $600 \mathrm{MU} / \mathrm{min}(0.629$ $\mathrm{Gy} / \mathrm{min})$. This figure indicates that the normalized dose undergo a linear relationship in this range with a high regression coefficient $\left(\mathrm{R}^{2}=0.929\right)$.

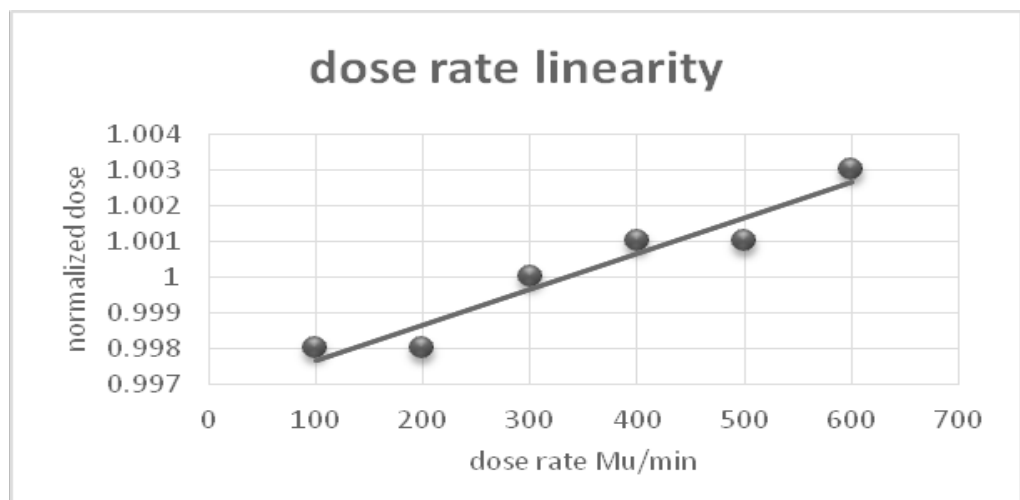

Fig. 4.3 the dose rate linearity between $100 \mathrm{MU} / \mathrm{min}$ and $600 \mathrm{MU} / \mathrm{min}$.

\section{Dose linearity}

Figure 4.4 shows that the relation between measured dose at the detector and the MUs was linear for doses above 1MU (0.007Gy).The regression coefficient was almost $1\left(\mathrm{R}^{2}=0.9999\right)$.

\section{Output factors (field size)}

Figure 4.5 shows that the output measurements increases with the field size

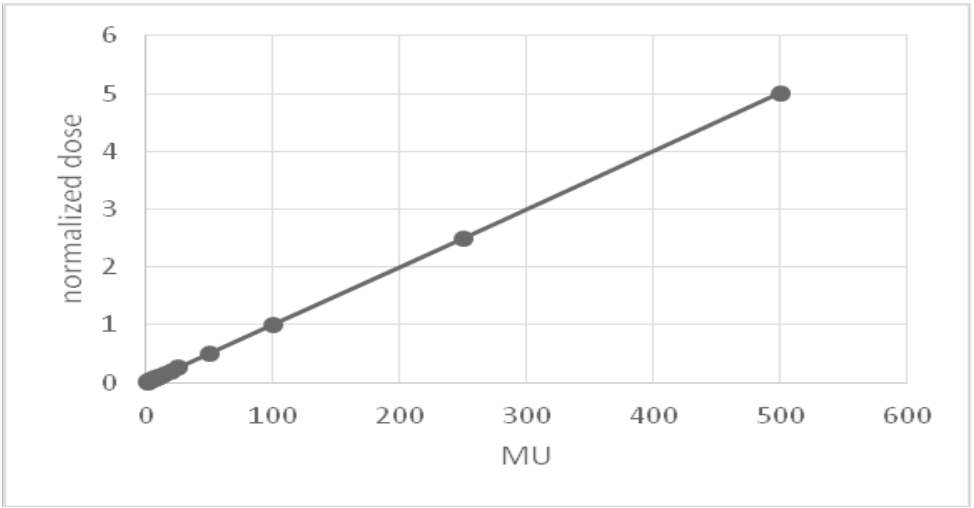

Fig. 4.4. The linearity of the dose for the 729 detector array

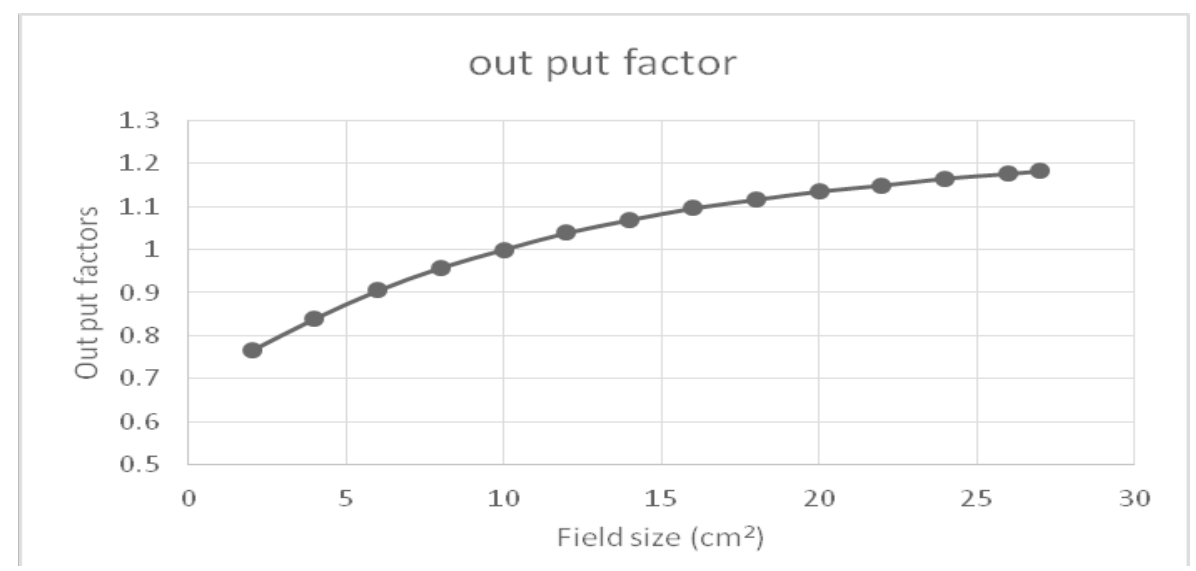

Fig. 4.5. The output factors measurements for the detector array 
The reconstruction algorithm accuracy

The gamma passing rate for the $10 \times 10 \mathrm{~cm}^{2}$ arc and the $10 \times 10 \mathrm{~cm}^{2}$ single static field were $99.2 \&$ $98.5 \%$, which mean that the difference is $0.7 \%$ and this indicates that the cylindrical phantom moves with acceptable synchrony with the gantry rotation as shown in Fig. 4.6.

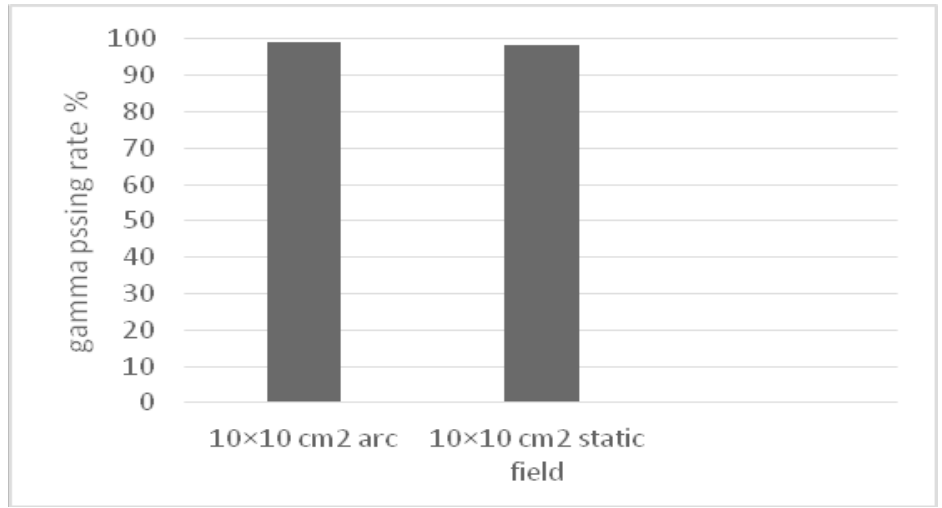

Fig. 4.6.The cylindrical phantom moves synchronously with the linac

\section{The accuracy of the algorithm}

The TG (target - gun) and LR (left - right) profiles taken across the measured dose distribution image were compared to that calculated by the treatment planning system for a $10 \times 10 \mathrm{~cm}^{2}$ beam. This measured and calculated profiles were in good agreement with some deviation in the penumbra region as shown in figures $(4.7 \& 4.8)$. The gamma pass rate was $99.2 \%$.The rotation allows detectors to measure at many angles and the average measured value by the detectors will smear out the resolution effect. This can be shown in Fig. 4.11.

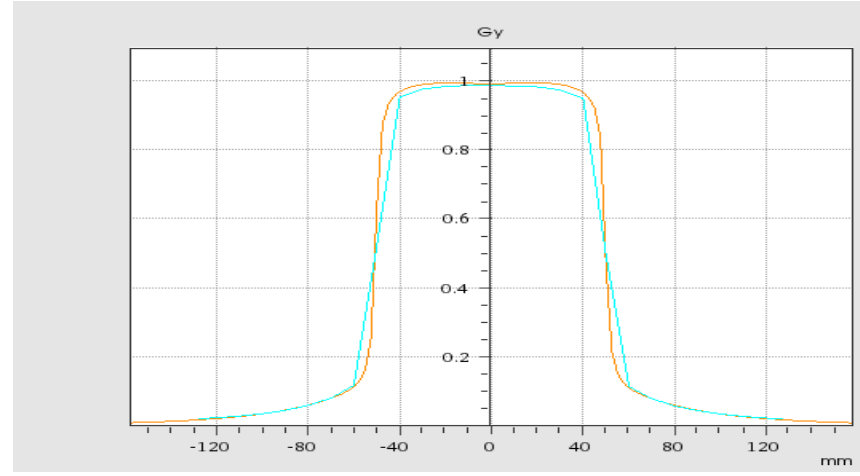

Fig.4.7. Static field $10 \times 10 \mathrm{~cm} 2$, coronal plane isocentric cross $L R$ profile: Octavius $4 \mathrm{~d}$ with 729 detector array (blue curve versus TPS (orange curve).

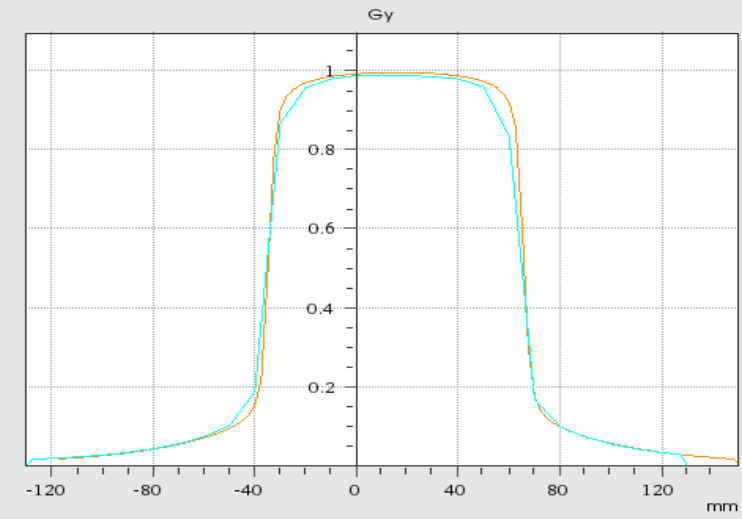

Fig.4.8.Static field $10 \times 10 \mathrm{~cm} 2$, coronal plane isocentric cross TG profile: Octavius $4 \mathrm{~d}$ with 729 detector array (blue curve versus TPS (orange curve)

Egypt. J. Biophys. Biomed. Eng., Vol. 18 (2017) 
Clinical IMRT and VMAT plans delivery

The verification of 26 IMRT and VMAT plans of different cases (Head and Neck, Brain, Breast and Prostate) showed that there is a high agreement between the 3D dose reconstructed by the Octavius system and the eclipse treatment planning system according to the gamma index factor. The highest agreement is in the prostate plans followed by brain, breast and finally H\&N cases, but also, there are failed voxels resulting from the high dose gradient regions (the regions adjacent to the critical organs and on the field edges) of the measured clinical plans in the different cases as shown in figure $4.9 \& 4.10$. The passed voxels in VMAT plans are higher than those of IMRT plans for the same cases and the reason will be discussed later.

The dose line profiles of the calculated image by the eclipse planning system and the measured one by the Octavius show good agreement with some mismatching in the field edges (the shoulders) as shown in Figure 4.11\&4.12. This mismatching was found to be dependent on the dose rate as can be seen in figure 4.13. We plotted the absolute dose difference between a measured Octavius line profile for a $10 \times 10 \mathrm{~cm}^{2}$ and the treatment planning dose line profile as a function of the dose rate. It was shown that the absolute dose difference decrease with the increase of the dose rate from 100 till $600 \mathrm{MU} / \mathrm{min}$. The mean of gamma passing rate were $98.0 \%, 95.5 \%, 96.2 \%$ and $93.3 \%$ for IMRT plans of prostate, breast, brain and Head

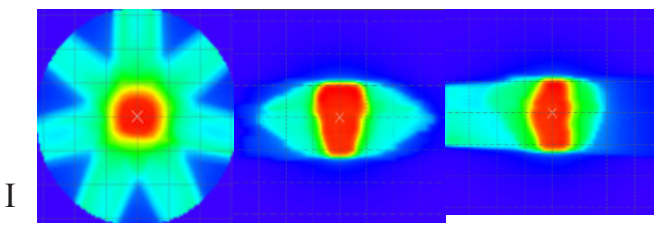

II
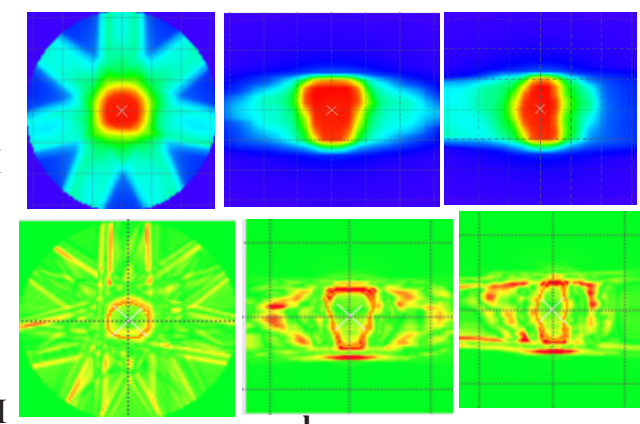

a

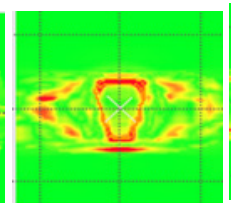

b

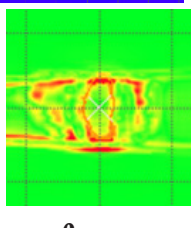

c
Fig. 4.9 (I) eclipse and (II)Octavius calculated dose matrices in a. axial, b. coronal and c. sagittal planes of IMRT plan for prostate case .(III) Gamma analysis results for the three planes. and Neck cases respectively. Also the mean of gamma passing rate were $99.4 \%, 98.1 \%, 98.6 \%$ and $96.3 \%$ for VMAT plans of prostate, breast, brain and Head and Neck cases, respectively. This was calculated using global 3D gamma common criterion $3 \% / 3 \mathrm{~mm}$ and passing threshold value $>95 \%$. This shows that the gamma index results of the VMAT plans verifications in all different cases of different tumor's locations are higher than those of IMRT plan and the least gamma passing rate was for IMRT plans of head and neck cases that couldn't achieve the passing threshold value.

\section{Test the sensitivity of the gamma criteria}

The average of gamma passing rate using the criteria $4 \% / 4 \mathrm{~mm}$ for IMRT plans of $\mathrm{H} \& \mathrm{~N}$ cases was $>95 \%$ but for the other criteria $(2 \% / 2 \mathrm{~mm}$, $3 \% / 3 \mathrm{~mm}, 3 \% / 4 \mathrm{~mm}$ and $4 \% / 3 \mathrm{~mm})$ were $<95 \%$ .The $4 \% / 4 \mathrm{~mm}$ criteria achieves the threshold passing value as shown in Figure 4.14.

Comparison between the stationary and the rotational Octavius measurements

The mean gamma pass rate measured by the stationary Octavius phantom for the IMRT plans is $97.4 \%$ and it is higher than those measured by the rotational Octavius phantom in all the cases $95 \%$, with the $\mathrm{P}$ value $=0.024421$. This can be ascribed to the effect of couch attenuation which can be estimated to be $2.6 \%$, as the gamma passing rate for the static anterior field was $98.3 \%$ and for the posterior static field was $95.7 \%$.
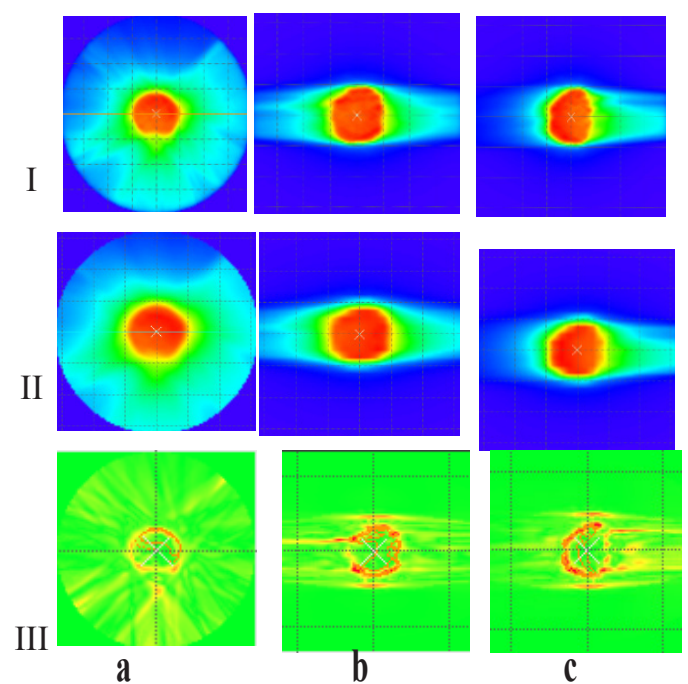

Fig.4.10.(I) eclipse and (II)Octavius calculated dose matrices in a. axial, b. sagittal and c. coronal planes of VMAT plan for prostate case . (III) Gamma analysis results for the three planes.

Egypt. J. Biophys. Biomed. Eng., Vol. 18 (2017) 


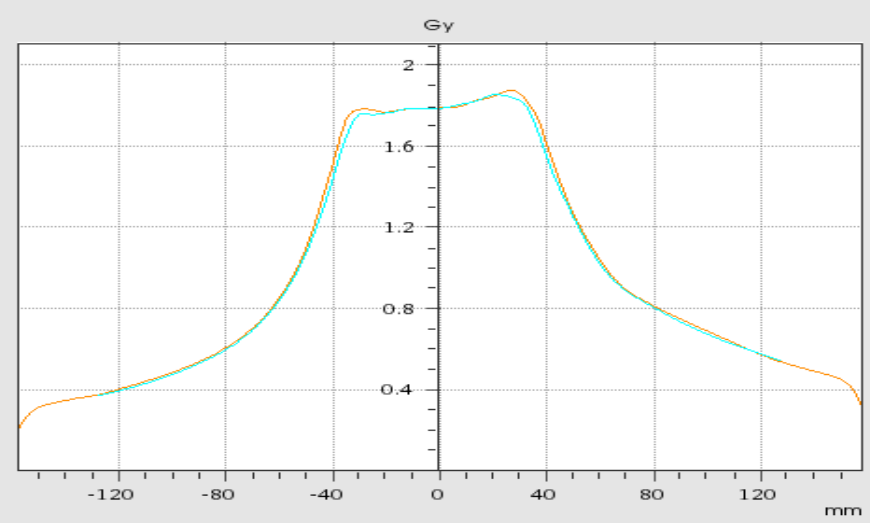

Fig.4.11: Dose profile lines taken across both the calculated and measured dose distribution at LR direction for VMAT plan of prostate case delivered using $\mathrm{DR}=600 \mathrm{Mu} / \mathrm{min}$.

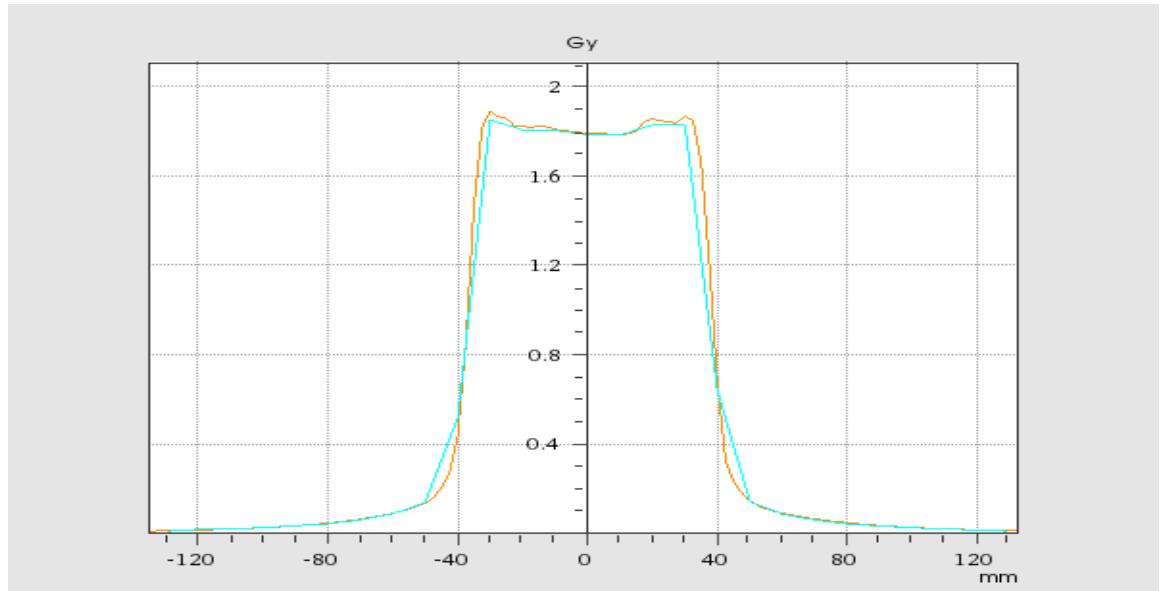

Fig.4.12 Dose profile lines taken across both the calculated and measured dose distribution at TG direction for VMAT plan of prostate case.

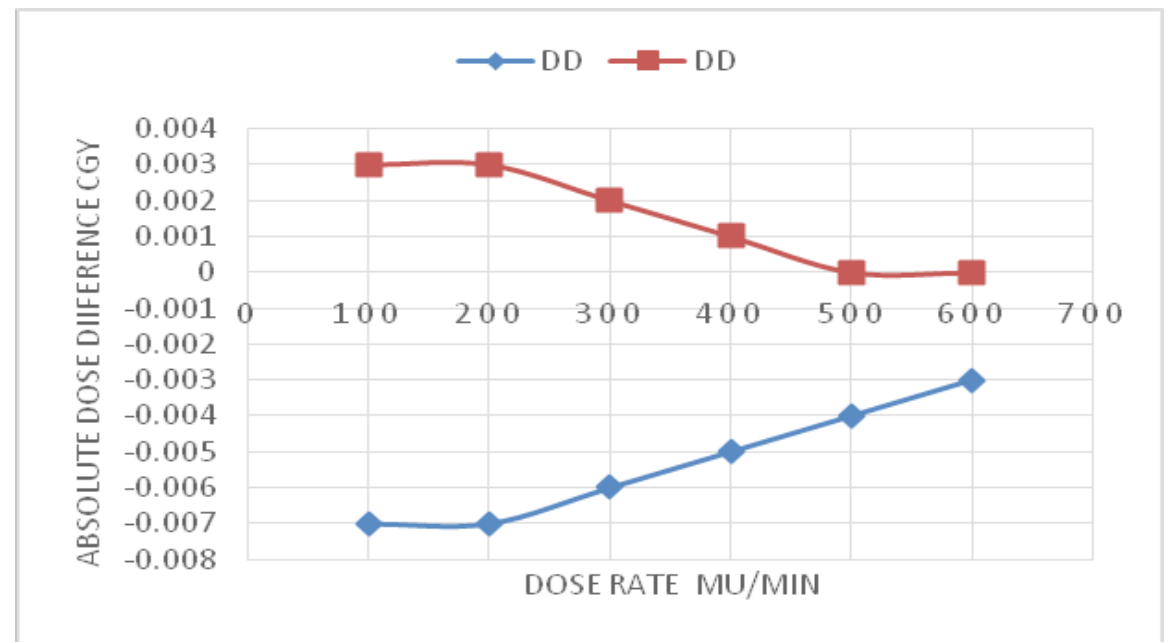

Fig. 4.13.The effect of the dose rate on the absolute dose difference of the LR line dose profiles (the measured one and the calculated one) for a static $10 * 10 \mathrm{~cm}$

Egypt. J. Biophys. Biomed. Eng., Vol. 18 (2017) 


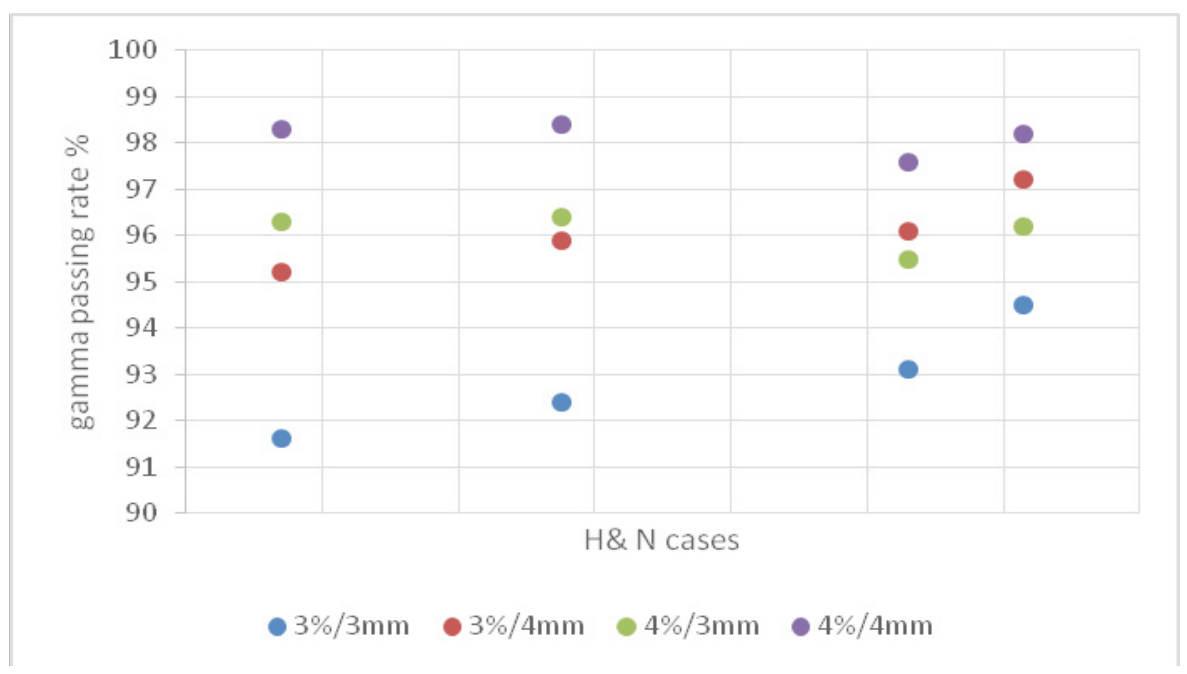

Fig. 4.14.The average of gamma passing rate for the various gamma index criteria of clinical IMRT plans of H\&N cases

Factors affect the gamma passing rate

The complexity

The gamma pass rate is $99.9 \%, 97.7 \%$ and $84.0 \%$ for plan one (plan with prescribed dose for all the targets $=50 \mathrm{~Gy}$ ), plan two (the same plan with prescribed dose $=50 \mathrm{~Gy}$ and $70 \mathrm{~Gy}$ ) and plan three (the same plan with different prescribed doses 50, 60 and 70Gy). The steep dose gradient in plan three resulted in a reduction of the gamma passing rate.

\section{Field sizes}

Figure 4.15 shows that there is no certain trend for the gamma index using the common criteria $3 \% / 3 \mathrm{~mm}$ with different field sizes.

\section{The prescribed dose}

Figure 4.16 shows that the gamma index doesn't vary with different prescribed doses.

\section{The effect of collimation rotation}

Figure 4.17 shows the gamma index calculated using the common criteria $3 \% / 3 \mathrm{~mm}$ for the different plans. The gamma passing rate was always smaller with the zero collimation plans. Rotating the collimator angle from $0^{\circ}$ to $45^{\circ}$ increases the area of irradiated detectors and improves the spatial resolution and thus the number of passed voxels and the gamma index increase.

Increasing number of fields

Increasing number of the fields of the plan result in increasing the gamma index as shown in Figure 4.18.

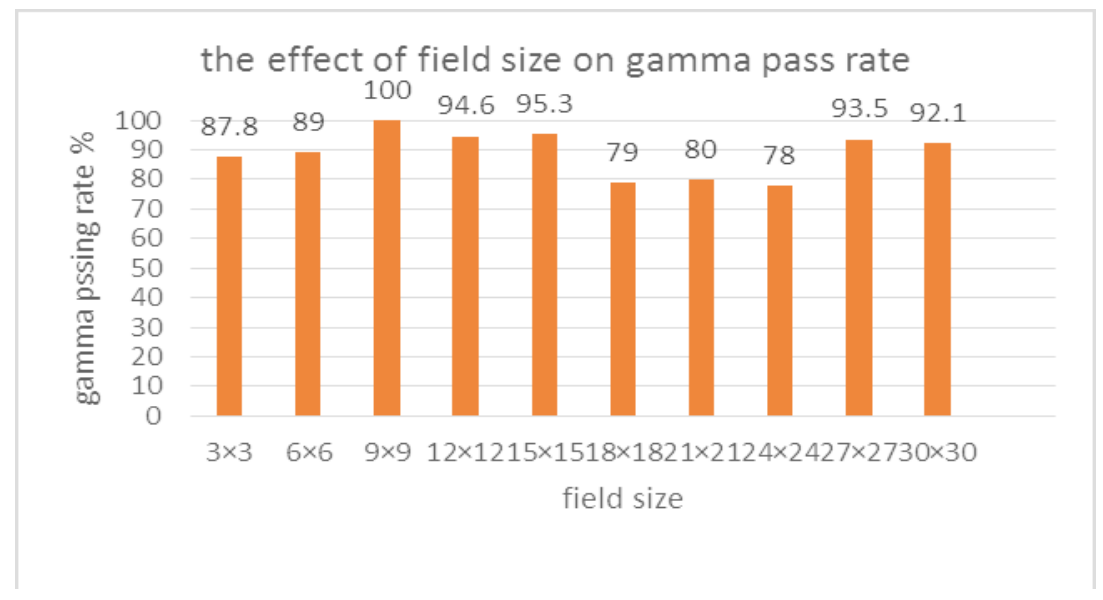

Fig. 4.15: the effect of field sizes on gamma passing rate. 


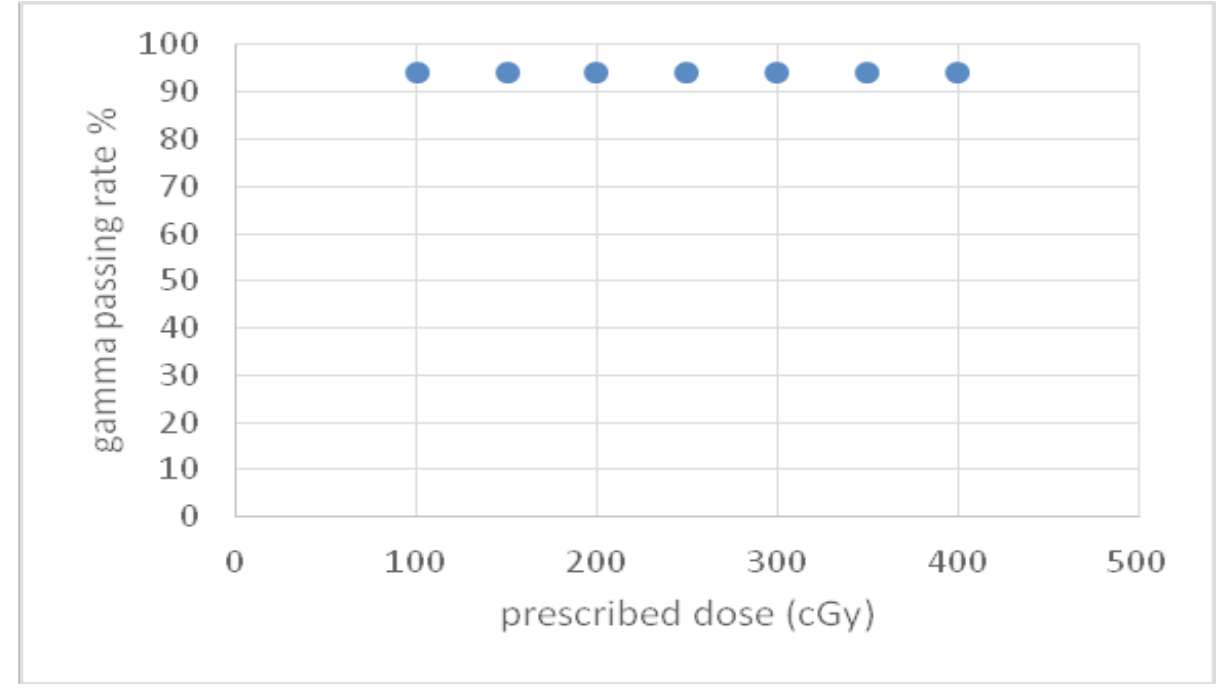

Fig 4.16. The different prescribed dose doesn't affect the gamma passing rate

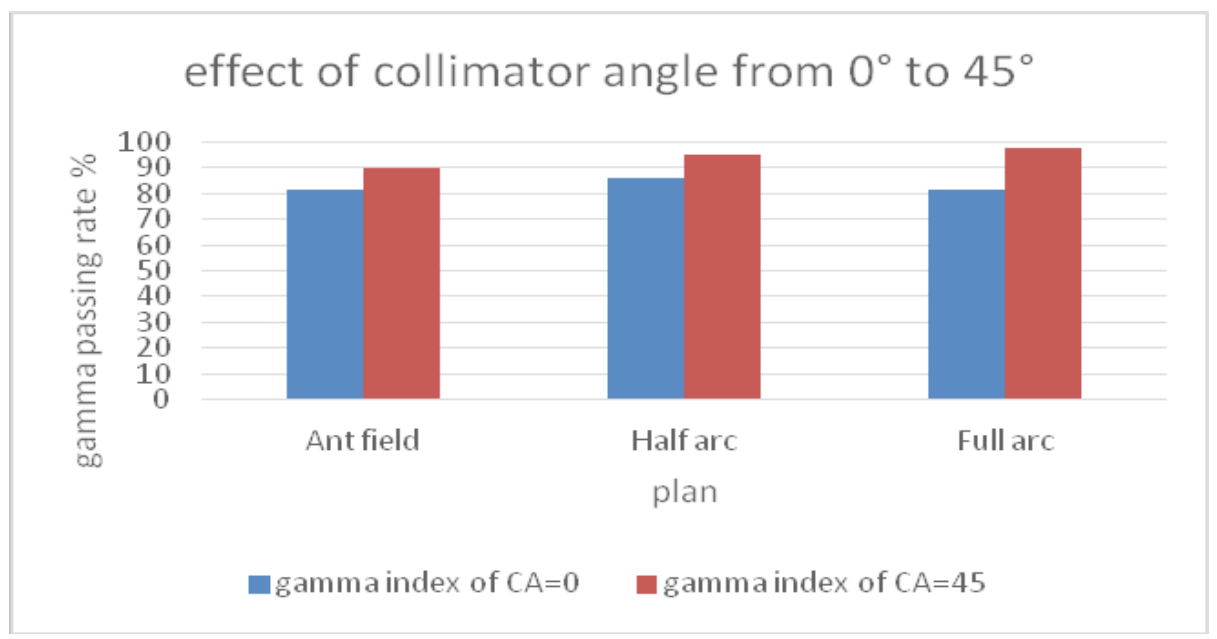

Fig. 4.17. The effect of collimator angle rotation on the gamma pass rate.

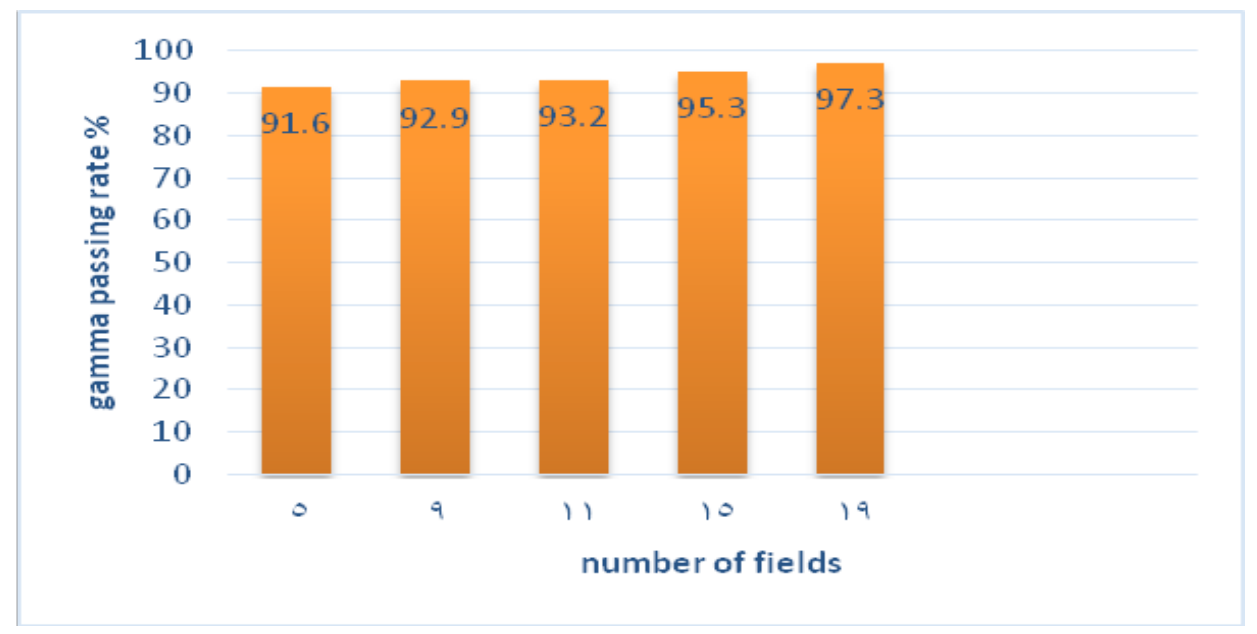

Fig.4.18.The effect of increasing number of fields on gamma index

Egypt. J. Biophys. Biomed. Eng., Vol. 18 (2017) 


\section{Discussion}

The Octavius $4 \mathrm{D}$ measuring system together with the 729 detector array is an adequate tool for advanced techniques verification and it is dosimetrically and mechanically stable.A dose warm -up is required to ensure the stability of the detector response and it is estimated to be around $800 \mathrm{MU}$ and this agree with Herzen et al., $2007^{(21)}$. The dose rate response and the dose measurements down to $1 \mathrm{MU}$ undergo a linear relationship with regression coefficient $(\mathrm{R}=0.992$ and 0.999$)$ and the detector array can estimate the dose till 0.007 Gy. The output factors measurements show a similar trend by increasing the field sizes from $1 \times 1 \mathrm{~cm}^{2}$ to $27 \times 27 \mathrm{~cm}^{2}$ and this agree with Mcgarry et al., 2013 ${ }^{(18)}$. The algorithm which depends on the PDD were accurate in the verification and the calculating of gamma index using the verisoft 5.1 with gamma passing rate $=99.2 \%$, this agree with Allgaier et al., 2013 ${ }^{(24)}$. The phantom was moving in a synchronizing motion with the gantry angle and the difference between the anterior arc and the anterior static field was $0.7 \%$.

The Octavius $4 \mathrm{D}$ measuring system with its detector array is a suitable system in verification of the dose distribution for IMRT and VMAT plans for different clinical cases in the three planes transverse, sagittal and coronal plane not only in the measured plane and this is an obvious advantage as some authors like Stasi et al., 2012 (23) showed deficiencies in detecting the errors using single detector array plane.

There is a high agreement between the 3D dose reconstructed by the Octavius system and the eclipse treatment planning system according to the gamma index factor, But also, there are failed voxels resulting from the high dose gradient regions (the regions adjacent to the critical organs and on the field edges) of the measured clinical plans in the different cases and in penumbra regions due to poor resolution and large active volume of the detectors in the array and this agree with Calvo et al., $2012^{(19)}$.

The dose lines profiles show a good agreement between the calculated image from the eclipse treatment planning system and the measured one by the Octavius 4D measuring system with some mismatching in the penumbra region at the field edges (shoulders) and it is found that this mismatching in the in the shoulders of the profiles decrease by increasing the dose rate from $100 \mathrm{MU} /$ $\min$ to $600 \mathrm{Mu} / \mathrm{min}$ and also due to the relatively larger detector size and its resolution. This agree with Allgaier et al., $2013^{(24)}$

The gamma passing rate and the succeeded voxels for the VMAT plans is higher than those of IMRT plans for all the cases. This can be explained by our results for the effect of rotating the collimator angle. The collimation angle used in VMAT increases the detector array space and the resolution and this agree with Mcgarry et al., $2013^{(18)}$, Another reason is the homogeneity index of VMAT plans which was higher than those of IMRT plans thus decreasing the dose gradient and this is in agreement with Amin et al., $2017^{(25)}$.

The gamma passing rate of the IMRT plans for head and neck cases showed the least value and couldn't achieve the threshold passing value $>95 \%$ using the common criteria $3 \% / 3 \mathrm{~mm}$. The QA sensitivity was tested for different criteria (2\%/2mm, $3 \% / 3 \mathrm{~mm}, 3 \% / 4 \mathrm{~mm}, 4 \% / 3 \mathrm{~mm}$ and $4 \% / 4 \mathrm{~mm})$. the gamma passing rate was highest with the $4 \% / 4 \mathrm{~mm}$ and this agree with Hussein et al.,2013 ${ }^{(5)}$ and Calvo et al., 2012 $2^{(19)}$. It is found based on our studied head and neck patients that it can be hard to achieve our passing threshold for criteria less than $4 \% / 4 \mathrm{~mm}$ for this kind of cases.

The couch factor estimated to be $2.6 \%$ and this was result in reduction of the gamma pass rate. This agree with Mcgarry et al., 2013 ${ }^{(18)}$. It is found that the gamma index factor is affected by the complexity which mean that increasing the dose gradient, decreases the gamma passing rate and this agree with Allgaier et al., 2013(24). Increasing the number of the fields will improve the homogeneity of the dose distribution, decrease the dose gradient and hence increase the gamma index value and this can add another reason for why the gamma index of VMAT plan is higher than those of IMRT plan. However different field sizes and changing the prescribed dose doesn't affect the gamma index.

\section{Conclusion}

The Octavius 4D measuring system together with the 729 detector array is validated and characterized for $6 \mathrm{MV}$. A warm up is required to ensure the stability of the detector. This system is accurate dosimetrically and mechanically. The Octavius 4D measuring system is a suitable tool in the dosimetric verification of the IMRT and VMAT plans for the different cases 


\section{References}

1. Bortfeld T., IMRT., a review and preview. Phys Med Biol.; 51(13), R363-R379 (2006).

2. P.Dixon and B.O'Sullivan ., Radiotherapy quality assurance: time for everyone to take it seriously. European Journal of Cancer; 39 (4), 423-429 (2003).

3. Ebert MA, Harrison KM, Cornes D, Howlett SJ, JosephDJ and Kron T., ComprehensiveAustralasian multicentre dosimetric intercomparison: issues, logistics and recommendations. $J$ Med Imaging Radiat Oncol; 53,119-31(2009).

4. B. Fraass, K. Doppke, M. Hunt, G. Kutcher, G. Starkschall, R. Stern and J. Van Dyke : Quality assurance for clinical radiotherapy treatment planning. The international Journal of medical physics research and practice; 25(10),1773-1829

5. Mohammad Hussein, Elizabeth J. Adams, Thomas J. Jordan, Catharine H. Clark and Andrew Nisbet: A critical evaluation of the PTW 2D-ARRAY seven29 and OCTAVIUS II phantom for IMRT and VMAT verification. Journal Of Applied Clinical Medical Physics, 14(6), 274-292(2013).

6. Otto K. Volumetric modulated arc therapy: IMRT in a single gantry arc. Med Phys, 35(1),31017(2008)

7. Teoh M, Clark CH, Wood K, Whitaker S, Nisbet A: Volumetric modulated arc therapy: a review of current literature and clinical use in practice. $\mathrm{Br} J$ Radiol, 84(1007),967-96(2011).

8. Carver A, Gilmore M, Riley S, Uzan J, Mayles P: An analytical approach to acceptance criteria for quality assurance of Intensity Modulated Radiotherapy. Radiother Oncol, 100(3), 45355(2011).

9. Feygelman V, Zhang G, Stevens C, Nelms BE: Evaluation of a new VMAT QA device, or the "X" and "O" array geometries. J Appl Clin Med Phys, 12(2),3346(2011).

10. Masi L, Casamassima F, Doro R, Francescon P: Quality assurance of volumetric modulated arc therapy: evaluation and comparison of different dosimetric systems. Med Phys, 38(2),61221(2011)

11. G. M. Mancuso, J. D. Fontenot, J. P. Gibbons, and B. C. Parker: Comparison of action levels for patient-specific quality assurance of intensity modulated radiation therapy and volumetric modulated arc therapy treatments. Med. Phys. 39(7), 4378-4385 (2012).

12. M. Bakhtiari, L. Kumaraswamy, D. W. Bailey, S. de Boer, H. K. Malhotra, and M. B. Podgorsak, :Using an EPID for patient-specific VMAT quality assurance.Med. Phys. 38(3),1366-1373 (2011).

13. E. Spezi, A. L. Angelini, F. Romani, and A. Ferri:Characterization of a 2D ion chamber array for the verification of radiotherapy treatments. Phys.Med. Biol. 50(14),3361-3373 (2005).

14. A. Van Esch, C. Clermont, M. Devillers, M. Iori, and D. P. Huyskens: On-line quality assurance of rotational radiotherapy treatment delivery by means of a 2D ion chamber array and the Octavius phantom. Med. Phys.34(10),3825 -3837 (2007).

15. V. Chandraraj, S. Stathakis, R. Manickam, C. Esquivel, S. S. Supe, and N.Papanikolaou: Comparison of four commercial devices for RapidArc and sliding window IMRT QA. J. Appl. Clin. Med. Phys. 12(2), 338-349(2011).

16. A. Gago-Arias, L. Brualla-González, D. M. González-Castaño, F. Gómez,M. S. García, V. L. Vega, J. M. Sueiro, and J. PardoMontero:Evaluation of chamber response function influence on IMRT verification using 2D commercial detector arrays. Phys. Med. Biol. 57(7), 2005-2020(2012).

17. Y. Shimohigashi, F. Araki, H. Tominaga, J. Sakata, K. Kawasaki, N. Kanetake,Y. Iwashita, S. Yoshimura, T. Kawakami, T. Ishihara, T. Okuda, and K. Kogo: Angular dependence correction of MatriXX and its application to composite dose verification. J. Appl. Clin. Med. Phys. 13(5), 198214(2012).

18. Conor K Mcgarry, Barry O'Connell and Denise Irvine: Octavius 4D characterization for flattened and flattening filter free rotational deliveries. Med. Phys. 40 (9), 091707-11 (2013)

19. O. Calvo ,S. Stathakis, A. N. Gutiérrez, C. Esquivel and N. Papanikolaou: 3D Dose Reconstruction of Pretreatment Verification Plans Using Multiple 2D Planes from the OCTAVIUS/Seven29 Phantom Array. Technology in Cancer Research and Treatment.11(1),69-82 (2012)

20. B. Poppe, A. Ruehmann, K. Willborn, B. Allgaier, and D. Harder:Three dimensional gamma-index analysis and considerations of the reference level definition for dosimetric IMRT plan verification with 2D ionization chamber arrays. IFMEB 
Proceedings on World Congress on Medical Physics and Biomedical Engineering .25(1), 248249 (2009)

21. J. Herzen, M. Todorovic, F. Cremers, V. Platz, D. Albers, A. Bartels, and R. Schmidt:Dosimetric evaluation of a $2 \mathrm{D}$ pixel ionization chamber for implementation in clinical routine. Phys. Med. Biol, 52(4), 1197-1208(2007).

22. M. Stasi, S. Bresciani, A. Miranti, A. Maggio, V. Sapino, and P. Gabriele:Pretreatment patientspecific IMRT quality assurance: A correlation study between gamma index and patient clinical dose volume histogram. Med. Phys, 39(12), 76267634 (2012).
23. B. Allgaier, E. Schüle and J. Würfel: Dose reconstruction in the OCTAVIUS 4D phantom and in the patient without using dose information from the TPS. PTW-Freiburg Physikalisch-Technische Werkstätten Dr. Pychlau GmbH Lörracher Straße Freiburg, Germany, 7 (2013).

24. Amin El. A., El-Sayed M . El ., Abdelsattar M. S. , Somaia M. El . and Noha A. H . : Physical Evaluation and Verification of Different Radiotherapy Techniques for Prostate Cancer. The International Journal of Science Technoledge, 5(5), 58-70 (2017).

إن تقنيات العلاج بالاشعاع المتطوره مثل تقنية العلاج بالاشعاع متغير الثدة و تقنية العلاج القوسى الحجمى إنى

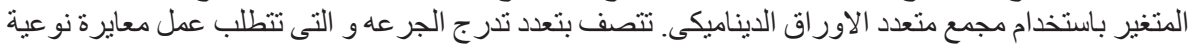

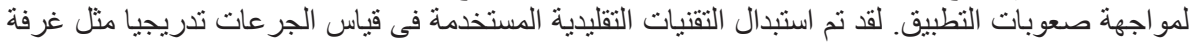

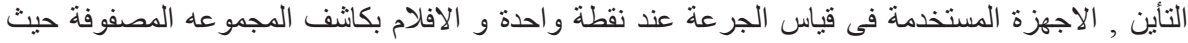

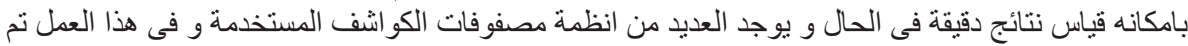

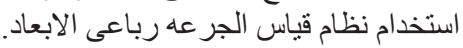

الغرض من الرسالة هو توصيف و تقييم نظام الاوكتافيوس رباعى الابعاد و صحة استخدامه في قياس

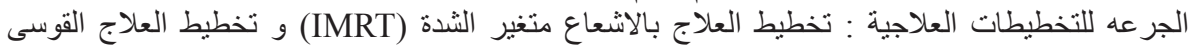

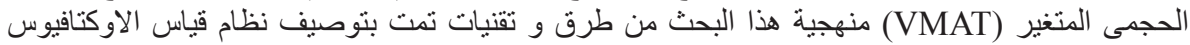

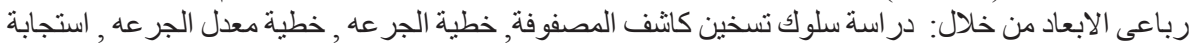

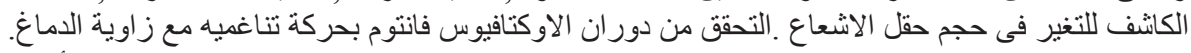

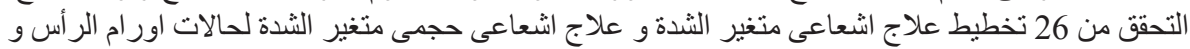

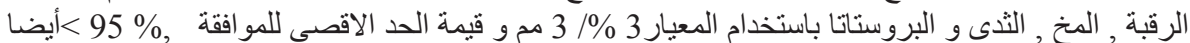

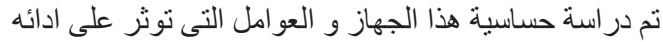

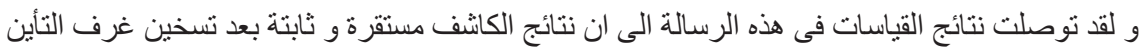

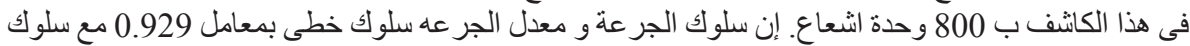

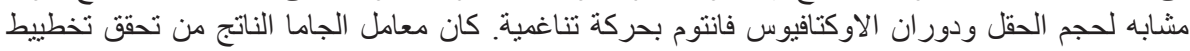

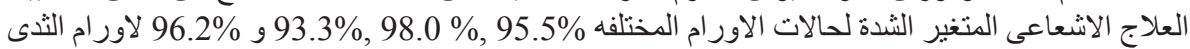

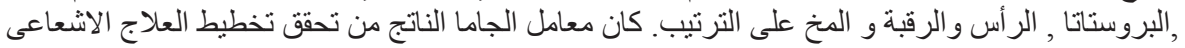

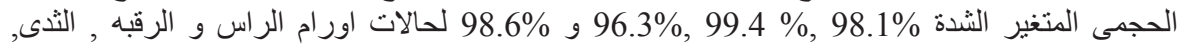

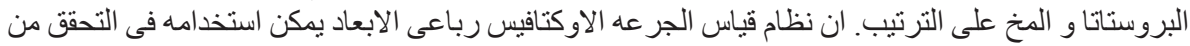

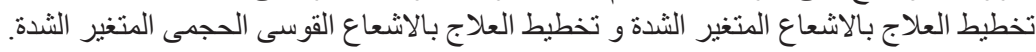

توصى نتائج العمل بالرسالة بامكانية تحسين الاداء باستخدام كاثف مصفوفة ذو دقة مكانية أعلى من المستخدم حاليا.

Egypt. J. Biophys. Biomed. Eng., Vol. 18 (2017) 